\title{
Cervico-Facial Actinomycosis: Epidemiological and Clinical Comments
}

\author{
${ }^{1}$ Silvia Tortorici, ${ }^{1}$ Francesco Burruano, ${ }^{1}$ Maria Lidia Buzzanca, \\ ${ }^{1}$ Paolo Difalco, ${ }^{2}$ Cabibi Daniela and ${ }^{2}$ Maresi Emiliano \\ ${ }^{1}$ Department of Oral Surgery, University of Palermo, Italy \\ ${ }^{2}$ Department of Patology, University of Palermo, Italy
}

\begin{abstract}
In a retrospective investigation performed in the department of Surgical Odontostomatology of Palermo University Polyclinic from 1997-2006, the authors examined 12 cases of cervico-facial actinomycosis, taking into consideration age range, gender distribution, predisposing factors and symptoms. In concordance with reports in literature, they found that the disease was perimandibular in $65 \%$, they also report the diagnostic methods and therapeutic approaches used in the study.
\end{abstract}

Key words: Cervicofacial bacterial tumour, skin fistula, actinomycosis, differential diagnosis

\section{INTRODUCTION}

Actinomycosis is a chronic infectious disease, caused by an anaerobic Gram-plus germ belonging to the Actinomyces family, which provokes a chronic productive and colliquative inflammatory reaction known as actinomycotic granuloma, with macroscopically-visual yellowish granules produced by bacterial proliferation. It is a rare disease occurring in three different clinical forms-cervico-facial, thoracic and abdominal.

The cervico-facial site is the most common of the three types and it is found in about $50 \%$ of the cases, it is also known as lumpy jaw and involves both the soft and hard tissue of the head and neck region. The various infection sites may regard the scalp, the paranasal sinuses, the palate, the parotid gland, the temporal bone, the tear glands, the cheeks, the lower jaw, the tongue, the larynx and the lower pharyn $x^{[1,2]}$.

The aim of our study was to define the diagnostic algorhythm of cervico-facial actinomycosis by drawing attention to its differential features compared with other diseases showing similar ulcerative and infiltrating clinical aspects.

\section{MATERIALS AND METHODS}

A retrospective investigation conducted in the department of Surgical Odontostomatology of Palermo University Polyclinic from 1997-2006, led to the diagnosis of 12 cases of cervico-facial actinomycosis in patients ranging from 16-71 years of age (mean 45.67 years) and made up of 7 men (58\%) and 5 women $(42 \%)$.
Ten patients presented syringoid skin lesions (Fig. 1A), one 20-year-old woman patient had a nodular swelling at the III median of the dorsum linguae (Fig. 1B), one male patient of 60 had a osteolytic lesion of the left premolar mandible.

The following predisposition factors were identified from the patients' medical history:

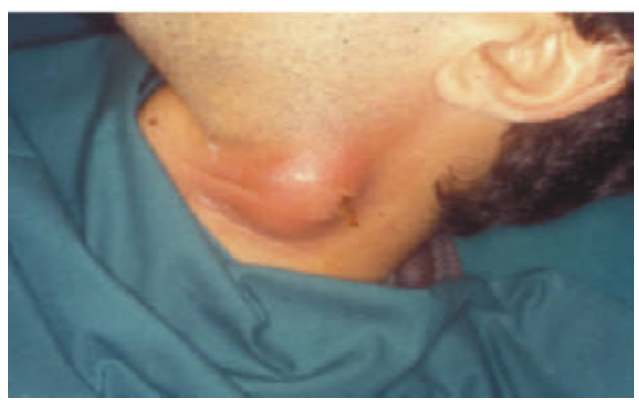

Fig. 1A: Swelling with clear signs of inflammation and initial fistolization

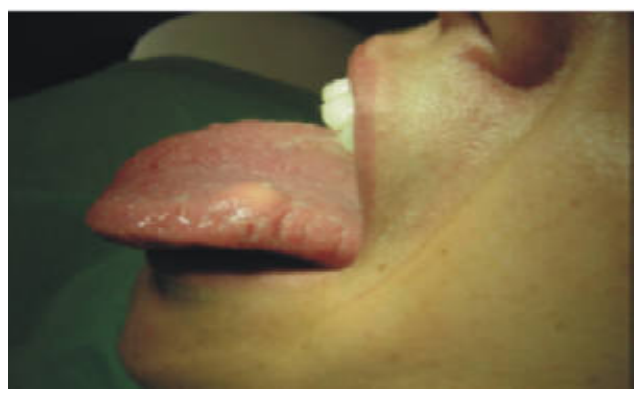

Fig. 1B: At the III median of the dorsum linguae, the typical actinomycotic nodule 
- Four patients had previously undergone tooth extraction

- Four patients had deep dental decay

- Two patients had parodontal pockets

- No particular cause was identified in the remaining patients

The patients presenting fistulas were affected by edema, swelling of the soft tissues with the formation of skin abscesses and general symptoms such as fever and weight loss. One patient was affected by lockjaw. In 8 patients $(66.6 \%)$ the swelling was painful, while in the remaining subjects it was not.

In concordance with literature ${ }^{[1,3]}$ we found that the perimandibular region was the most frequent disease site ( $65 \%$ of cases). In no case was there lymph node involvement.

All the patients underwent an X-ray examination and anatomo-pathological analysis involving desquamative or fine-needle aspiration cytology, either by means of the F.N.A.B. method or by exudate sampling.

\section{RESULTS AND DISCUSSION}

Etiology: Actinomycetes are a group of bacteria divided into six subgroups: israelii, naeslundii, viscosis, odontolyticus, meyeri and pyogenes.

Actinomyces Israelii is the most common form found in human beings, it was first described in Israel in 1878 and is a slow-growing, anaerobic Gram-plus bacterium. It was formerly classified for a considerable time as a fungus because of its filamentous structure, but is in fact a Gram-positive bacterium with a very particular aspect, made up of a club-shaped corpus with branches, which stains with Gomori methenamine silver $^{[3,4]}$.

Actinomyces are a normal part of the gastrointestinal flora, they have also been isolated in saliva, salivary calcoli, carious cavities, bone sequestra and tonsillar crypts - all areas where the detergent action of saliva is less effective, creating a condition of insufficient oxygenation. They are not generally pathogenic, but any continual lesion of the mucosa may lead to infection of the surrounding tissues, especially in patients who are already in poor health or immunodepressed. A negative condition of the oral cavity is also considered a predisposing factor to the disease.

Actnomycosis is more commonly found in adult males. In the cervico-facial form, the entry point of the bacterium is generally a decayed tooth, this results in the formation of an infectious, torpid granuloma which gets larger and larger, with eventual bone damage and possible suppuration and the formation of abscesses and fistulas $^{[1,5]}$.

The thoracic form affects the lungs after the aspiration of oral secretions ${ }^{[6]}$, while the abdominal form is presumably due to the perforation of a diverticulum or of the appendix or during a trauma ${ }^{[7]}$.

Symptoms: The incubation period ranges from two months to a year. In the cervico-facial form, a hard, painless swelling with unclear margins forms in the soft tissues, in time, this becomes fluctuating and small, multiple, communicating abscesses surrounded by granulation tissue are formed (Fig. 1C), the purulent material contains yellowish sulfurous granules (Fig. 1D). The infectious process spreads to the surrounding tissues such as the pharynx, the tongue, the saliva glands, the jaw bones, with resulting osteitis, or the skin, forming fistulas in various points, sometimes, but not often, the infection may enter the blood stream and cause a general form which may be fatal.

When the skin surface is involved, there is a painless rubescence, with fluctuating zones alternating with hard, wooden areas, there may be one or more syringoid openings. If no surgical action is taken, the fistulas tend to heal and new openings may form. This process gives rise to retraction of the scar tissue, deformation of the skin and esthetic damage (Fig. 1E).

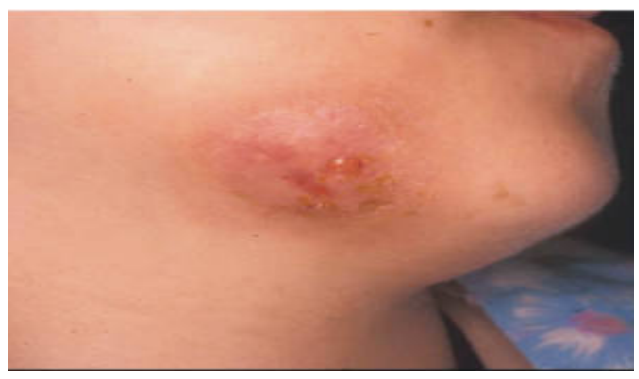

Fig. 1C: A hard, only slightly painful swelling covered with skin showing multiple ulceration

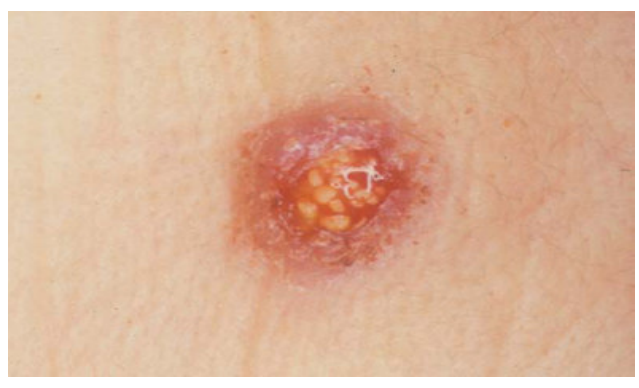

Fig. 1D: Ulceration producing pus containing yellowish granules 


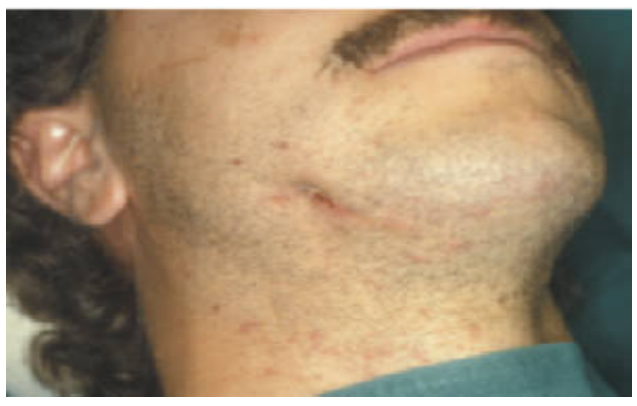

Fig. 1E: Scar tissue leading to skin damage

The clinical variants of the cervico-facial form are:

- Chronic osteitis,

- Osteolytic lesion with granulation tissue which presents as polycystic at X-ray examination ${ }^{[8]}$

- On the tongue (3\% of cases), with the formation of a hard nodule attached to the muscular layer ${ }^{[9]}$

- At the pterygomaxillary site with lockjaw

- As a parapical or paradental abscess ${ }^{[10,11]}$

- The regional lymph nodes are rarely involved and if so, without pain ${ }^{[12]}$

Histological aspect: The main element is an actinomycotic granuloma, made up of a network of threads and claviform formations, the peripheral tissue is granulomatous and full of plasma-cells, fibroblasts, giant-cells and blood vessels, there is also an infiltrate of polymorphonucleates.

An actinomycotic granuloma may develop into a purulent form with several fistulas in the case of multiple granulomas or into a productive form with fibrosis.

Diagnosis: The osteo-cutaneous fistula simulates an odontogenic abscess, especially when the clinical picture has been altered by an improper use of antibiotics, furthermore, the presence of sulfurous granules visible to the naked eye, should suggest the presence of an actinomycosis.

Diagnosis is based not only on the symptoms, but also on the identification of Actinomyces Israelii in the siero-purulent sputum or in the biopsy specimens. Fineneedle aspiration is a useful sampling method in order to avoid contamination with aspecific microbic flora ${ }^{[13]}$ and shows up the threads bunched up in balls or tufts (Fig. 1F) ${ }^{[14]}$. Another useful diagnostic method is an actinomyces culture on glioglycolate and on agarglucose $^{[15,16]}$.

This diagnostic work-up is essential for a differential diagnosis compared with other ulcerative

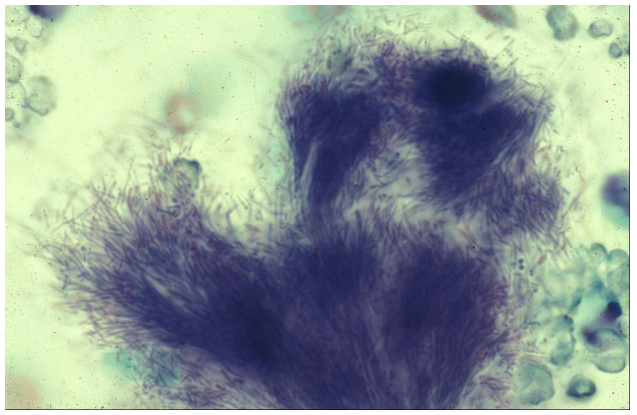

Fig. 1F: Microscopic examination shows fine bacterial threads in bundles looking typically like tufts of hair.Papanicolau stain: overall magnification $400 \mathrm{x}$

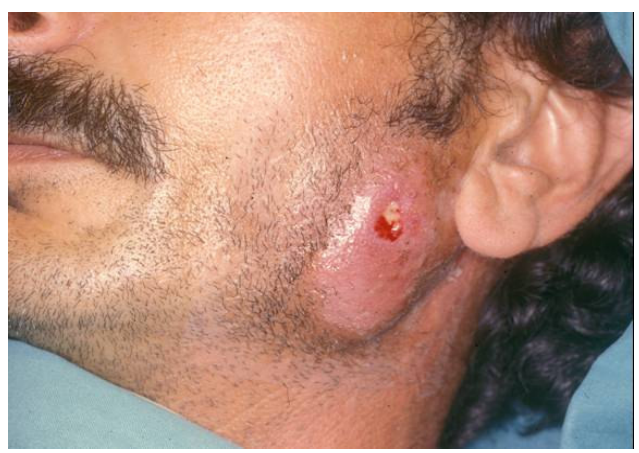

Fig. 1G: Skin fistula of ulcerated parotid carcinoma shows a hard swelling with an ulcerated surface and raised, hardened margins

diseases or infiltrative masses of the jaw ${ }^{[17]}$. A CT scan and MRI are not sufficient for distinguishing actinomycosis from malignant tumoral masses ${ }^{[18,19]}$.

A biopsy should be performed when a small alveolar carcinoma lesion is found together with massive infiltration of the surrounding tissues, in such cases the presence of actinomyces may complicate the situation $^{[20]}$. It is also essential to perform a histological examination in cases where a parotid carcinoma includes a hard swelling which simulates an actinomycotic indurative mass and which may develop into a skin fistula (Fig. 1G), in such cases the regional lymph nodes are not always involved ${ }^{[21]}$.

Diagnostic doubts are also necessary in cases of oral tuberculosis and of ulcerated syphilitic gumma.

Prognosis and treatment: The disease develops slowly with a favorable prognosis in the cervico-facial forms but less so in the thoracic, abdominal and generalized forms, especially when the CNS is involved, but in all cases early diagnosis is always recommended. 
Surgery is always the best therapeutic approach towards cervico-facial actinomycosis, the infected tissues should be removed by means of ample curettage and drainage of the fistulas, together with subsequent antibiotic treatment ${ }^{[22,23]}$. This latter should consist of high doses of penicillin G (10-20,000,000 U/die IV) for several days or even weeks, followed by oral doses of penicillin $\left(2-4 \mathrm{~g} \mathrm{die}^{-1}\right)$ for from three to 12 months ${ }^{[1,12]}$. In subjects who are allergic to penicillin, tetracycline, erythromycin, clindamycin or cephalosporin are valid alternatives $^{[24]}$.

\section{CONCLUSIONS}

In spite of the fact that the disease presents a favorable prognosis in the great majority of cases, cervico-facial actinomycosis tends to involve several tissues and organs in the area, with the resulting functional and esthetic damage. In order to guarantee a favorable disease outcome, an early diagnosis and adequate therapy are absolutely essential. Furthermore, it should be borne in mind that symptoms of actinomycosis may be microbically superimposed on slow-developing malignant lesions. A biopsy sample should therefore be taken even when symptoms clearly point to an actinomycosis and especially in the presence of a purulent exudate containing the typical sulfurous granules.

In cases where the clinical picture mainly involves skin fistulas, plastic surgery should be performed only when it is certain that the disease is in remission.

\section{REFERENCES}

1. Miller, M. and A.J. Haddad, 1998. Cervicofacial actinomycosis. Oral Surg. Oral Med. Oral Pathol. Radiol. Endod., 85: 496-508.

2. Belmont, M.J., P.M. Behar and M.K. Wax, 1999. Atypical presentations of actinomycosis. Head and Neck, 21: 264-268.

3. Russo, T.A., 2000. Agents of Actinomycosis. In: Mandell, Douglas and Bennett's Principles and Practice of Infectious Disease. Mandell, G.L., J.E. Bennett and R. Dolin (Eds.). Churchill Livingstone, New York, pp: 2645-2654.

4. Henderson, H.M. and S.W. Chapman, 2003. Infections due to Fungi, Actinomyces and Nocardia. In: Reese and Betts' a Practical Approach to Infectious Diseases. Betts, R.F., S.W. Chapman and R.L. Pen (Eds.). Lippincott Williams and Wilkins, Philadelphia, pp: 588-648.

5. Neville, B.W., D.D. Damm, C.M. Allen and J.E. Bouquot, 1995. Oral and Maxillofacial Pathology. WB Saunders Company, Philadelphia, pp: 156-157.
6. Kwong, J.S., N.L. Muller, J.D. Godwin, D. Aberle and M.R. Grymaloski, 1992. Thoracic actinomycosis: CT findings in eight patients. Radiology , 183: 189-192.

7. Ha, H.K., H.J. Lee, H. Kim, H.J. Ro, Y.H. Park, S.J. Cha and K.S. Shinn, 1993. Abdominal actinomycosis: CT findings in 10 patients. Am. J. Roentgenol., 161: 791-794.

8. Bartkowski, S.B., J. Zapala, P. Heczkoand and M. Szuta, 1998. Actinomycotic osteomyelitis of the mandible: review of 15 cases. J. Cranio Maxillof. Surg., 26: 63-67.

9. Lavilla Martin De Valmaseda, M., F. Benitez Camacho, M. Garcia Jimenez, J.L. Sanchez-Jara Sanchez, R. De Saa Alvarez, M.A. Gonzalez and B. Garcia Garcia, 2000. Lingual actinomycosis: Diagnostic problems. Rev. Literat. Acta Otorrinolaringol. Esp., 51: 80-84.

10. Nagler, R.M., Y. Ben-Arieh and D. Laufer, 2000. Case report of regional alveolar bone actinomycosis: A juvenile periodontitis-like lesion. J. Periodontol., 71: 825-829.

11. Hirshberg, A., I. Tsesis, Z. Metzger and I. Kaplan, 2003. Periapical actinomycosis: A clinicopathologic study. Oral Surg. Oral Med. Oral Pathol. Oral Radiol. Endod., 95: 614-620.

12. Bennhoff, D.F., 1984. Actinomycosis: Diagnostic and therapeutic considerations and a review of 32 cases. Laryngoscope, 94: 1198-1217.

13. Silverman, J.F., 1991. Guides to Clinical Aspiration Biopsy: Infectious and Inflammatory Diseases and other Nonneoplastic Disorders. IGAKU-SHOIN Medical Publishers, Inc., New York, pp: 49-51,183.

14. Custal-Teixidor, M., J. Trull-Gimbernat, G. Garijo-López and M. Valldosera-Rosello, 2004. Fine-needle aspiration cytology in the diagnosis of cervicofacial actinomycosis: Report of 15 cases. Med. Oral Patol. Oral Cir. Bucal., 9: 464-470.

15. Von Lichtenberg, F., 1989. Infectious Disease. In: Robbins Pathologic Basis of Disease, Contran, R.S., V. Kumar and S.L. Robbins (Eds.). Saunders, Philadelphia, pp: 383-384.

16. Nagler, R., M. Peled and D. Laufer, 1997. Cervicofacial actinomycosis: A diagnostic challenge. Oral Surg. Oral Med. Oral Pathol. Oral Radiol. Endod., 83: 652-656.

17. Lewandowski, B. and A. Biskup, 1999. Difficulties in diagnosis of facial and perimaxillary tissue actinomycosis. Pol. Merkur Lekarski., 7: 277-278.

18. Weber, A.L. and A. Siciliano, 2000. CT and MR imaging evaluation of neck, infections with clinical correlations. Radiol. Clin. North Am., 38: 941-968. 
19. Park, J.K., H.K. Lee, H.K. Ha, H.Y. Choi and C.G. Choi, 2003. Cervicofacial actinomycosis: CT and MR imaging findings in seven patients. AJNR Am. J. Neuroradiol., 24: 331-335.

20. Ferrada, C.R., D. Oddo, L.V. Ferrada, I. Palacios and L. Ristori, 1998. Actinomicosis associada a tumores malignos cervicofaciais. Rev. Ch. Infectol., 5: 41-43.

21. Lang-Roth, R., C. Schippers and H. Eckel, 1998. Cervical actinomycosis. A rare differential diagnosis of parotid tumor HNO. 46: 354-358.
22. Dokic, M., V. Begovic, S. Loncarevic, R. Vulovic, J. Dimitrijevic, S. Popovic and R. Aleksic, 2004. Actinomycosis: A multidisciplinary approach to a clinical problem. Vojnosanit Pregl., 61: 315-319.

23. Oostman, O. and R.A. Smego, 2005. Cervicofacial Actinomycosis: Diagnosis and Management. Curr. Infect. Dis. Rep., 7: 170-174.

24. Martin, M.V., 1985. Antibiotic treatment of cervicofacial actinomicosis for patients allergic to penicillin: A clinical and in vitro study. Br. J. Oral Maxillof. Surg., 23: 428-434. 